\title{
Neutron charge radius from intrinsic quark flavour generation
}

\section{Trinhammer, Ole L.}

\section{Published in:}

Epl

Link to article, DOI:

10.1209/0295-5075/134/61002

Publication date:

2021

\section{Document Version}

Publisher's PDF, also known as Version of record

Link back to DTU Orbit

\section{Citation (APA):}

Trinhammer, O. L. (2021). Neutron charge radius from intrinsic quark flavour generation. Epl, 134(6), [61002]. https://doi.org/10.1209/0295-5075/134/61002

\section{General rights}

Copyright and moral rights for the publications made accessible in the public portal are retained by the authors and/or other copyright owners and it is a condition of accessing publications that users recognise and abide by the legal requirements associated with these rights.

- Users may download and print one copy of any publication from the public portal for the purpose of private study or research.

- You may not further distribute the material or use it for any profit-making activity or commercial gain

- You may freely distribute the URL identifying the publication in the public portal

If you believe that this document breaches copyright please contact us providing details, and we will remove access to the work immediately and investigate your claim 
LETTER • OPEN ACCESS

\section{Neutron charge radius from intrinsic quark flavour generation}

To cite this article: Ole L. Trinhammer 2021 EPL 13461002

View the article online for updates and enhancements.
You may also like

- The quantum group, Harper equation and
$\frac{\text { structure of Bloch eigenstates on a }}{\text { honevcomb lattice }}$
M Eliashvili, G I Japaridze and G
Tsitsishvili
- On proton charge radius definition
Ole L. Trinhammer and Henrik G. Bohr
- ABJ triality: from higher spin fields to
Strings
Chi-Ming Chang, Shiraz Minwalla, Tarun
Sharma et al.




\title{
Neutron charge radius from intrinsic quark flavour generation
}

\author{
Ole L. Trinhammer ${ }^{(a)}$ \\ Department of Physics, Technical University of Denmark - Fysikvej bld. 307, DK-2800 Kongens Lyngby, Denmark
}

received 8 January 2021; accepted in final form 30 March 2021

published online 3 September 2021

\begin{abstract}
The finite, non-zero mean square neutron charge radius is understood in the present work to have a topological origin from an intrinsic neutron configuration in the Lie group $U(3)$. We introduce up and down quark orbits in the configuration for the neutron mass eigenstate. From reciprocal Gaussian curvatures we infer a mean square charge radius of -0.1075 square fermis a few standard deviations away from the world average of -0.1161 square fermis.

open Cáccess Copyright (c) 2021 The author(s)

Published by the EPLA under the terms of the Creative Commons Attribution 4.0 International License (CC BY). Further distribution of this work must maintain attribution to the author(s) and the published article's title, journal citation, and DOI.
\end{abstract}

Introduction. - Determinations of the mean square neutron charge radius has been under long discussion [1] until a certain level of authoritative values were reached $[2,3]$. However, the question has recently regained interest [4-6]. The value $\left\langle r_{n}^{2}\right\rangle=(-0.1161 \pm$ $0.0022) \mathrm{fm}^{2}$ cited by the Particle Data Group, PDG [7], just manages to connect new investigations which state $\left\langle r_{n}^{2}\right\rangle=\left(-0.106_{-0.005}^{+0.007}\right) \mathrm{fm}^{2},\left(-0.105_{-0.006}^{+0.005}\right) \mathrm{fm}^{2}[4,5]$ and $\left\langle r_{n}^{2}\right\rangle=\left(-0.122 \pm 0.004_{(\text {stat })} \pm 0.010_{(\text {sys })}\right) \mathrm{fm}^{2}$ [6]. The new results lie on opposite sides of the uncertainty interval of [7] with the PDG value based primarily on $[2,3,8]$. The uncertainties may be underestimated or the theoretical backgrounds behind the different methods may not be fully compatible. After submission Heacock et al. found $\left\langle r_{n}^{2}\right\rangle=(-0.1100 \pm 0.0092) \mathrm{fm}^{2}$ by interferometry [9].

The basic idea in the present theoretical work is to interpret the mean square radius as a manifestation of an intrinsic curvature in the neutron configuration. It yields $\left\langle r_{n}^{2}\right\rangle=(-0.1075 \pm 0.0020) \mathrm{fm}^{2}$, a value that results from reciprocal Gaussian curvatures of $u$ and $d$ quark tracks on the intrinsic configuration space, the Lie group $U(3)$. The $u$ quark tracks have positive curvature on the average and the $d$ quark tracks have negative curvature. It should be stressed that our conception of quarks is as generated by scattering on baryons rather than as fundamental fields constituting baryons [10].

In earlier works the mean square charge radius is derived from the concept of a neutron-electron scattering length $b_{n e}$. This scattering length contribution is then

\footnotetext{
(a) E-mail: ole.trinhammer@fysik.dtu.dk (corresponding author)
}

transformed to $\left\langle r_{n}^{2}\right\rangle$ by the relation [3]

$$
\left\langle r_{n}^{2}\right\rangle=\frac{3 m_{e} a_{0}}{m_{n}} b_{n e}
$$

where $m_{e}$ is the electron mass, $m_{n}$ is the neutron mass and $a_{0}$ is the Bohr radius [7].

One way to determine $b_{n e}$ (and actually the first one used [11]) is to scatter thermal neutrons on a contained gaseous target. The scattering will then result from the incoming neutrons interacting with atoms in the target. The atoms consist of both electrons and nuclei. The hard problem is to extract the minute interaction of a possible intrinsic neutron charge distribution with the electric fields in the atoms from the much larger strong interaction between the incoming neutrons and the atomic nuclei in the target. It has even been discussed whether the effect should be ascribed to weak interactions [12]. Around 1986, however, the theoretical framework was more or less settled [1] and the contribution from the neutron-electron scattering length was grouped in a class of $0.1 \%$-level "corrections" to the measured total scattering lengths, whereas weak interaction terms were estimated to the vanishing level of $10^{-35}$ relative to the two leading contributions from strong interactions and from interaction of the incoming neutron magnetic dipole moment with the atomic magnetic moment [1].

As it became clear that the electric contribution is not vanishingly small —and its sign consistently was negative - its origin was interpreted as an intrinsic neutron charge distribution being negative on the outskirts of the neutron interior. Already from the beginning (virtual) mesons were introduced for this purpose [11] 


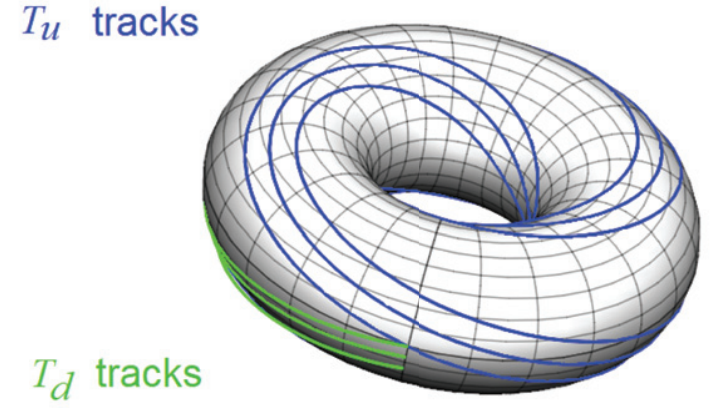

Fig. 1: Quark flavour tracks on the toroidal part of the intrinsic neutron configuration space, the Lie group $U(3)$. The Gaussian curvature is negative on the inner part of the torus. The torus embeds in the laboratory space two out of three dimensions of the Abelian part of $U(3)$. The two dimensions are excited by flavour generators $T_{u}, T_{d}$ (26) with integral curves [13] of the vector fields (blue and green, respectively) excited by the momentum form applied to the intrinsic neutron wave function as a ground state of (19).

and this interpretation is still alive in [14] which yields $\left\langle r_{n}^{2}\right\rangle=-0.119 \mathrm{fm}^{2}$ as a center value within the interval $(-0.13 \cdots-0.108) \mathrm{fm}^{2}$.

Quark models were introduced [15] using approximate harmonic oscillators for an interior neutron wave function of three flavour quarks, two negatively charged $d$ quarks and one positively charged $u$ quark. The $d$ quarks are more likely to be in a relative spin-one state suggested to give a repelling force driving the $d$ 's further from the center of mass. For a prior critique of quark model calculations not even giving the right sign, cf. [16]. The recent work [6] also relies on the quark concept, now by introducing quark flavour-dependent form factors for electrondeuteron scattering.

In the present work we introduce flavour quarks from an intrinsic neutron configuration and find $\left\langle r_{n}^{2}\right\rangle=(-0.1075 \pm$ $0.0020) \mathrm{fm}^{2}$ with the uncertainty from a purely theoretical calculation relying only on a length scale $\pi a=r_{e}$ set by the very accurate classical electron radius $r_{e}=$ $2.8179403262(13) \mathrm{fm}[7,17,18]$. We get our value for $\left\langle r_{n}^{2}\right\rangle$ from average curvatures of quark flavour tracks with negatively charged $d$ flavours giving negative curvature contributions which on the average more than outweigh the positive curvature contributions from $u$ flavours, see fig. 1 . The flavour tracks are unfolded from an intrinsic configuration for the neutron state, see table 1 . The accuracy can be improved by expansion of the intrinsic neutron wave function on higher-order contributions than those included for the results in table 1.

Before we describe our model, we sketch the theoretical foundation for the most common experimental techniques.

Scattering length and form factor towards $\left\langle r_{n}^{2}\right\rangle .-$ In this section we give a short presentation of the concept of scattering length and the concept of form factor. For a
Table 1: Expansion of the neutronic groud state on Slater determinants (33) with expansion coefficients for the most important contributions representing $99.4 \%$ of the probability. The last column shows the reciprocal Gaussian curvatures of the individual contributions. The expansion coefficients are found from a diagonalisation of the Hamiltonian in (19) by a Rayleigh-Ritz method [10,19] with 75 terms corresponding to $0 \leq p<r \leq N$ and $0<q \leq N$ with $N=5$.

\begin{tabular}{cccc}
\hline $\begin{array}{c}\text { Component } \\
p q r\end{array}$ & $\begin{array}{c}\text { Coefficient } \\
c_{p q r}\end{array}$ & $\begin{array}{c}\text { Probability } \\
c_{p q r}^{2}\end{array}$ & \multicolumn{1}{c}{$\mathrm{fm}^{2}$} \\
\hline 011 & 0.69342275 & 0.48083511 & -0.1072039923 \\
012 & 0.49859432 & 0.248596296 & -0.107371106 \\
013 & 0.05871873 & 0.003447889 & -0.1083696661 \\
021 & 0.23871329 & 0.056984035 & -0.1073274076 \\
022 & 0.12232589 & 0.014963623 & -0.1102520727 \\
023 & -0.01284764 & 0.000165062 & -0.1087722956 \\
112 & 0.41766359 & 0.174442874 & -0.1083344645 \\
113 & 0.04649025 & 0.002161343 & -0.1099410075 \\
122 & 0.11337581 & 0.012854074 & -0.1067497261 \\
Totals & - & 0.994450307 & -0.107501387 \\
$(N=3$ & - & 0.997013839 & $-0.107493495)$ \\
\hline
\end{tabular}

thorough description of electromagnetic neutron-atom interactions we recommend Sears [1] in which the different contributions to the scattering length are defined. For the extraction of the square of the charge radius from deuteron form factors we recommend Filin et al. [5]. For a textbook presentation of the relation between charge radius and form factor we recommend Sakurai [20]. We follow Sears and Sakurai for our resumes here.

Sears imagines an incoming plane wave beam of neutrons interacting with the scattering medium through a potential $V$ and leaving the medium again as scattered plane waves in various directions. He uses a Born approximation [21] to write down a scattering amplitude

$$
F=-\frac{m_{n}}{2 \pi \hbar^{2}} \int e^{-i \mathbf{p}^{\prime} \cdot \mathbf{r} / \hbar} V(\mathbf{r}) e^{i \mathbf{p} \cdot \mathbf{r} / \hbar} \mathrm{d} \mathbf{r},
$$

where $\mathbf{p}$ and $\mathbf{p}^{\prime}$ are the momenta of the incoming and outgoing neutrons, respectively. Sears writes the potential as a sum over four contributions,

$$
V=V_{\mathrm{N}}+V_{\mathrm{M}}+V_{\mathrm{E}}+V_{\mathrm{P}}
$$

where the first two terms are the large contributions mentioned in the introduction from strong interactions and the electromagnetic interaction from the neutron magnetic moment. The last two small terms are electromagnetic in origin with the third ascribed to an electrostatic energy arising from an intrinsic structure of the neutron and the fourth term arises from a possible electric polarisability of the neutron. Provided these potentials are independent on the neutron momentum, the integral in (2) may be written 
as a Fourier transform,

$$
F=-\frac{m_{n}}{2 \pi \hbar^{2}} V(\mathbf{q}), \quad V(\mathbf{q})=\int e^{i \mathbf{q} \cdot \mathbf{r} / \hbar} V(\mathbf{r}) \mathrm{d} \mathbf{r}
$$

with $\mathbf{q}=\mathbf{p}-\mathbf{p}^{\prime}$ being the momentum change of the scattering neutron and $\mathbf{r}$ is its varying position throughout the process. The linearity in (3) gives four scattering amplitudes to be considered individually from a theoretical point of view,

$$
F=F_{\mathrm{N}}+F_{\mathrm{M}}+F_{\mathrm{E}}+F_{\mathrm{P}}
$$

It turns out that all four terms are of a form to sum up as

$$
F=-b e^{i \mathbf{q} \cdot \mathbf{R} / \hbar}
$$

with $\mathbf{R}$ being the position of the atomic nucleus in the target and with $b$ being the total scattering length

$$
b=b_{\mathrm{N}}+b_{\mathrm{M}}+b_{\mathrm{E}}+b_{\mathrm{P}} .
$$

It is $b_{\mathrm{E}}$ which is of interest here ${ }^{1}$. It reads [1]

$$
b_{\mathrm{E}}=\frac{2 m_{n} Z e}{4 \pi \varepsilon_{0} \hbar^{2}}\left[\frac{z e}{q^{2}}-i \frac{l e}{q} \boldsymbol{\sigma} \cdot \frac{\mathbf{q}}{q}-\varepsilon\right]\left[1-f_{A}(\mathbf{q})\right], \quad q=|\mathbf{q}| .
$$

Here $f_{A}$ is a form factor for the atom. For vanishing neutron charge $z$ and vanishing neutron electric dipole length $l$ we have

$$
b_{\mathrm{E}}=-b_{\mathrm{I}} Z\left[1-f_{A}(\mathbf{q})\right]
$$

where

$$
b_{\mathrm{I}}=\frac{2 m_{n} e}{4 \pi \varepsilon_{0} \hbar^{2}} \varepsilon=\frac{1}{3} \frac{m_{n}}{m_{e}} \frac{\left\langle r_{n}^{2}\right\rangle}{a_{0}}
$$

in Sears' notation with $\varepsilon \equiv \frac{1}{6} e\left\langle r_{n}^{2}\right\rangle$ (cf. (18)) parametrising a neutron charge distribution term derived by Foldy $[1,22$, 23]. Sears calls $b_{\mathrm{I}}$ the intrinsic neutron-electron scattering length. We recognise the relation in (1) between $b_{n e}$ and the mean square charge radius $\left\langle r_{n}^{2}\right\rangle$.

The form factor $f_{A}(\mathbf{q})$ is a correction factor on the differential scattering cross-section of point particle scattering. It collects the effect of the target having an interior/intrinsic electric structure. One may think of unpolarised electrons scattering on atomic nuclei of atomic number $Z$ [20]

$$
\left(\frac{\mathrm{d} \bar{\sigma}}{\mathrm{d} \Omega}\right)=\left(\frac{\mathrm{d} \bar{\sigma}}{\mathrm{d} \Omega}\right)_{\text {point }}\left|f_{A}(\mathbf{q})\right|^{2} .
$$

For scattering on a point particle

$$
\left(\frac{\mathrm{d} \bar{\sigma}}{\mathrm{d} \Omega}\right)_{\text {point }}=\left(\frac{Z \alpha \hbar}{2 c}\right)^{2} \frac{E^{2}}{|\mathbf{p}|^{4} \sin ^{4}(\theta / 2)}\left(1-\beta^{2} \sin ^{2}(\theta / 2)\right)
$$

${ }^{1}$ The earliest data are expressed in interaction strengths as analogous depths $V_{0}$ (Fermi convention) [11] of corresponding radial square well potentials of range equal to the classical electron radius $r_{e}[17,18]$. These depths correspond to scattering lengths

$$
b_{n e} \approx \frac{1}{3} r_{e}^{3} \frac{2 m_{n}}{\hbar^{2}} V_{0} .
$$

See appendix A for a derivation of the conversion. with fine structure constant $\alpha$, electron energy $E$, scattering angle $\theta$ and $\beta=|\mathbf{p}| c / E$.

As mentioned, the form factor is interpreted as originating in a charge distribution $\varrho(\mathbf{x})$ in the target wherefore the Coulomb potential at distance $|\mathbf{x}|$ from the nucleus is substituted by an integral over the interior charge density

$$
-\frac{Z e}{4 \pi \epsilon_{0}|\mathbf{x}|} \rightarrow \frac{1}{4 \pi \epsilon_{0}} \int \mathrm{d}^{3} x^{\prime} \frac{\varrho\left(\mathbf{x}^{\prime}\right)}{\left|\mathbf{x}-\mathbf{x}^{\prime}\right|}
$$

which leads in the case of electron-neutron scattering to a neutron form factor [20]

$$
f_{n}(\mathbf{q})=\frac{1}{-Z e} \int \varrho(\mathbf{x}) e^{-i \mathbf{q} \cdot \mathbf{x} / \hbar} \mathrm{d}^{3} x
$$

In this interpretation thus, the form factor represents Fourier components of the charge distribution of the neutron target when in the scattering of an electron a momentum $\mathbf{q}=\mathbf{p}-\mathbf{p}^{\prime}$ is exchanged with the neutron.

For not too large momentum exchanges, the exponential in the form factor can be expanded,

$$
e^{-i \mathbf{q} \cdot \mathbf{x} / \hbar}=1-\frac{i \mathbf{q} \cdot \mathbf{x}}{\hbar}-\frac{1}{2}\left(\frac{\mathbf{q} \cdot \mathbf{x}}{\hbar}\right)^{2}+\cdots
$$

and for a spherical charge distribution, the expansion integrates to yield ${ }^{2}$

$$
f_{n}(\mathbf{q})=1-\frac{\left\langle r^{2}\right\rangle}{6 \hbar^{2}}|\mathbf{q}|^{2}+\cdots
$$

with $\left\langle r^{2}\right\rangle$ defining the mean square radius that is at our focus,

$$
\left\langle r^{2}\right\rangle=\int r^{2} \varrho \mathrm{d}^{3} x
$$

Now, what is determined experimentally is the form factor as of (12) leading (possibly through subtractions of other contributions as of (7)) to the electric scattering length as of (10). The results for $\left\langle r_{n}^{2}\right\rangle$ are derived from (17) and (11), respectively, based on the underlying interpretation.

From the experiments one cannot distinguish between the effect of an interior neutron charge distribution or an intrinsic neutron structure. We use the terms interior and intrinsic to represent, respectively, a spatial distribution within a certain (small, but finite) domain of laboratory space (the interior of a neutron) or a neutronic structure represented by an intrinsic configuration, i.e., a configuration excited at a point in laboratory space where the neutron is hit and the intrinsic configuration space "comes to life" in its entirety.

Baryon configurations. - We consider baryons as stationary states on an intrinsic configuration space, the

\footnotetext{
${ }^{2}$ It is customary to write the neutron form factor as a sum of electric and magnetic terms [6], but we shall not go into that here.
} 
Lie group $U(3)[24]^{3}$

$$
\frac{\hbar c}{a}\left[-\frac{1}{2} \Delta+\frac{1}{2} d^{2}(e, u)\right] \Psi(u)=\mathcal{E} \Psi(u), \quad u \in U(3) .
$$

The configuration space $U(3)$ contains all the three gauge groups of the standard model which motivates to use it for baryonic configurations; baryons namely feel all the three quantum interactions with gauge groups $S U(3)$ for the strong interactions and $U(1) \times S U(2)$ for the electroweak interactions. $U(3)$ has nine generators analogous to momentum, angular momentum and Laplace-RungeLenz operators in the laboratory space, which may explain its origin in scattering processes in the laboratory space which kinematically excite intrinsic degrees of freedom. The intrinsic degrees of freedom may be thought of as generalised spin. The electron spin was first realized as an intrinsic degree of freedom in 1926 by Uhlenbeck and Goudsmit [28]. We have our unitary configuration variable $u$ generated by a Hermitian operator $\chi$

$$
\begin{aligned}
& u=e^{i \chi}, \quad \chi=\theta_{j} T_{j}+\left(\alpha_{j} S_{j}+\beta_{j} M_{j}\right) / \hbar, \\
& j=1,2,3, \quad \theta_{j}, \alpha_{j}, \beta_{j} \in \mathbb{R} .
\end{aligned}
$$

Here the toroidal generators $T_{j},\left(e . g ., T_{2}=\operatorname{diag}(0,1,0)\right)$ equate the momentum generators $p_{j}$ in laboratory space

$$
i T_{j}=\frac{\partial}{\partial \theta_{j}}=\frac{a}{-i \hbar} p_{j}, \quad e^{i \theta_{j} T_{j}}=\operatorname{diag}\left(e^{i \theta_{1}}, e^{i \theta_{2}}, e^{i \theta_{3}}\right)
$$

the spin generators $S_{j}$ equate the angular momentum generators - in coordinate representation [29], $\left(x_{j}=a \theta_{j}\right)$

$$
S_{1}=a \theta_{2} p_{3}-a \theta_{3} p_{2}=\hbar \lambda_{7}=\hbar\left(\begin{array}{ccc}
0 & 0 & 0 \\
0 & 0 & -i \\
0 & i & 0
\end{array}\right),
$$

and the generators $M_{j}$ mix spin and flavour (25), e.g.,

$$
M_{1} / \hbar=\theta_{2} \theta_{3}+\frac{a^{2}}{\hbar^{2}} p_{2} p_{3}=\lambda_{6}
$$

The $M_{j}$ 's share commutators with quantum analogues of the Laplace-Runge-Lenz vector [30] $\mathbf{M}=c_{1}(\mathbf{p} \times \mathbf{L}-\mathbf{L} \times$ $\mathbf{p})+c_{2} / r \cdot \mathbf{r}$, which is a constant of motion in Kepler orbits and in the hydrogen atom [29]

$$
\left[M_{i}, M_{j}\right]=\left[S_{i}, S_{j}\right]=-i \hbar \varepsilon_{i j k} S_{k}
$$

Note the minus sign as in body fixed intrinsic coordinates for spin in nuclear physics.

The lambdas are the Gell-Mann matrices [29]. The spectrum of $\mathbf{S}^{2}$ is well-known [31] to be $s(s+1) \hbar^{2}$ with half odd integer $s$ in multiples of $\hbar$ for our intrinsic case. The spectrum of the positive definite $\mathbf{M}^{2}$ is [24]

$$
\mathbf{M}^{2} / \hbar^{2}=\frac{4}{3}\left(n+\frac{3}{2}\right)^{2}-s(s+1)-3-\frac{1}{3} y^{2}-4 i_{3}^{2},
$$

${ }^{3}$ This is a radical reinterpretation of the Kogut-Susskind Hamiltonian [25] of lattice gauge theory [26] using Manton's action [27] as a potential and taking $u$ to concern the full baryonic dynamics.

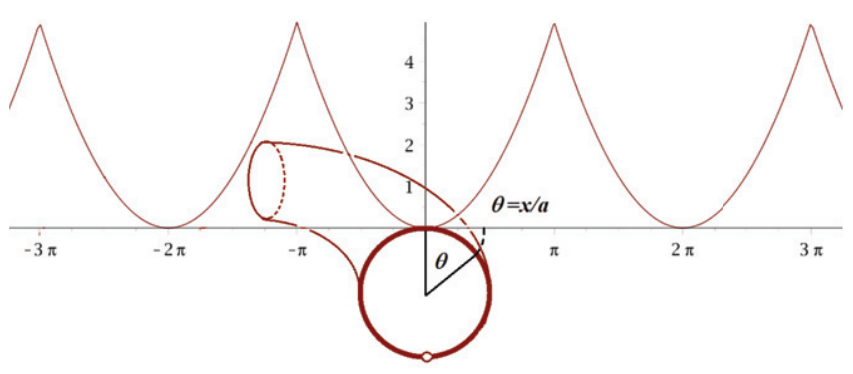

Fig. 2: The intrinsic potential is periodic in the dynamical eigenangles $\theta_{j}=x_{j} / a$. Figure from [10].

where $n$ is a non-negative integer, $y$ is baryonic hypercharge and $i_{3}$ is the three-component of baryonic isospin. To generate $u$ and $d$ flavour orbits we use

$$
T_{u}=\frac{2}{3} T_{1}-T_{3}, \quad T_{d}=-\frac{1}{3} T_{1}-T_{3}
$$

with quark charge coefficients on $T_{1}$ and common coefficients on $T_{3}$. The same generators have been used to derive $u$ and $d$ valence quark parton distributions for the proton [24] as well as the proton spin structure function and the proton magnetic dipole moment [10] in good agreement with experiments.

To solve (19) we first factorise the wave function

$$
\Psi(u)=\tau\left(\theta_{1}, \theta_{2}, \theta_{3}\right) \Upsilon\left(\alpha_{1}, \alpha_{2}, \alpha_{3}, \beta_{1}, \beta_{2}, \beta_{3}\right) .
$$

This is possible because the potential in (19) only depends on the eigenvalues $e^{i \theta_{j}}$ of $u$ [32] through the three dynamical eigenangles $\theta_{j}\left(\left[a \theta_{i}, p_{j}\right]=-i \hbar \delta_{i j}\right)$,

$$
\frac{1}{2} d^{2}(e, u)=\frac{1}{2} \operatorname{Tr} \chi^{2}=\sum_{j=1}^{3} w\left(\theta_{j}\right)
$$

with (fig. 2)

$w(\theta)=\frac{1}{2}(\theta-n \cdot 2 \pi)^{2}, \quad \theta \in[(2 n-1) \pi,(2 n+1) \pi], n \in \mathbb{Z}$.

To suit the potential we use a polar decomposition of the Laplacian on $U(3)$ [33]

$$
\Delta=\sum_{j=1}^{3} \frac{1}{J^{2}} \frac{\partial}{\partial \theta_{j}} J^{2} \frac{\partial}{\partial \theta_{j}}-\sum_{\substack{1 \leq i<j \leq 3 \\ k \neq i, j}} \frac{\left(S_{k}^{2}+M_{k}^{2}\right) / \hbar^{2}}{8 \sin ^{2} \frac{1}{2}\left(\theta_{i}-\theta_{j}\right)}
$$

where the van de Monde determinant [34]

$$
J=\prod_{1 \leq i<j \leq 3} 2 \sin \frac{1}{2}\left(\theta_{i}-\theta_{j}\right)
$$

We then introduce a measure scaled wave function

$$
\Phi(u)=J \Psi(u)=R \Upsilon .
$$

With (30) inserted, eq. (19) solves analogously to solving the hydrogen atom [21] by integrating over the off-toroidal 
degrees of freedom - now with three "radial" dimensions and $\Upsilon$ taking the role of spherical harmonics.

Since the labelling of the eigenangles is arbitrary, the toroidal wave function $\tau$ in (27) should be symmetric in these. The van de Monde determinant $J(31)$ is antisymmetric in the eigenangles and likewise will $R=J \tau$ have to be. We therefore expand $R$ on Slater determinants antisymmetric in the three toroidal eigenangles which we interpret as colour degrees of freedom. For unflavoured baryons of neutral charge we expand $R$ on the complete set

$$
f_{p q r}=\left|\begin{array}{ccc}
\cos p \theta_{1} & \cos p \theta_{2} & \cos p \theta_{3} \\
\sin q \theta_{1} & \sin q \theta_{2} & \sin q \theta_{3} \\
\cos r \theta_{1} & \cos r \theta_{2} & \cos r \theta_{3}
\end{array}\right|, \quad R=\sum_{p q r} c_{p q r} f_{p q r},
$$

where $p, q, r$ are integers $0 \leq p<r \in \mathbb{N}$ and $0<q \in \mathbb{N}$.

Flavour orbit curvatures towards $\left\langle r_{n}^{2}\right\rangle$. - In [10] we described how colour fields are generated from the intrinsic wave function by use of the exterior derivative, the momentum form [13]. We found quark colour fields in the laboratory space

$$
\psi_{j}(u)=\left.\mathrm{d} R_{u}\left(\partial_{j}\right) \equiv \frac{\mathrm{d}}{\mathrm{d} t} R\left(u e^{i t T_{j}}\right)\right|_{t=0}, \quad \partial_{j}=u i T_{j}
$$

and gluon fields

$$
G^{(k)}(u)=\mathrm{d} \Phi_{u}\left(\partial_{k}\right), \quad \partial_{k}=u \frac{i \lambda_{k}}{2}, \quad k=1,2, \cdots, 8
$$

to transform under the fundamental and the adjoint representation of $S U(3)$, respectively, and found that local gauge transformations in the laboratory space equate coordinate translations in the configuration space.

Similarly we generate the $u$ and $d$ quark flavour fields by derivation along the directions given in (26). We used this to derive $u$ and $d$ valence quark parton distributions for the proton in [24]. Here we focus on the neutron and consider the geometry of the orbits generated.

With two linearly independent generators in each of the two $T_{q}$ 's (26), their orbits will run along the same twodimensional torus, winding their helical ways in opposite orientation (fig. 1).

We want to ascribe curvatures with physical dimensions to these orbits. For that we use the length scale $a$ in (19). We determine the length scale from the classical electron radius $r_{e}[17,18]$ by taking $[24]$

$$
\pi a=r_{e} .
$$

Here the factor $\pi$ indicates a mapping to the flat laboratory space from the intrinsic torus ${ }^{4}$.

We now embed in the laboratory space the intrinsic torus on which $u$ and $d$ vector fields are excited according to the common coefficient $h=-1$ and the individual fractional quark charges $e_{q}=\frac{2}{3},-\frac{1}{3}$, respectively.

\footnotetext{
${ }^{4}$ In [24] we describe how the classical electron radius enters baryonic physics via the neutron to proton decay. We write on the creation of the electron charge in this decay as a "peel off" from the neutron, leaving a "scarred" nucleon: the proton.
}

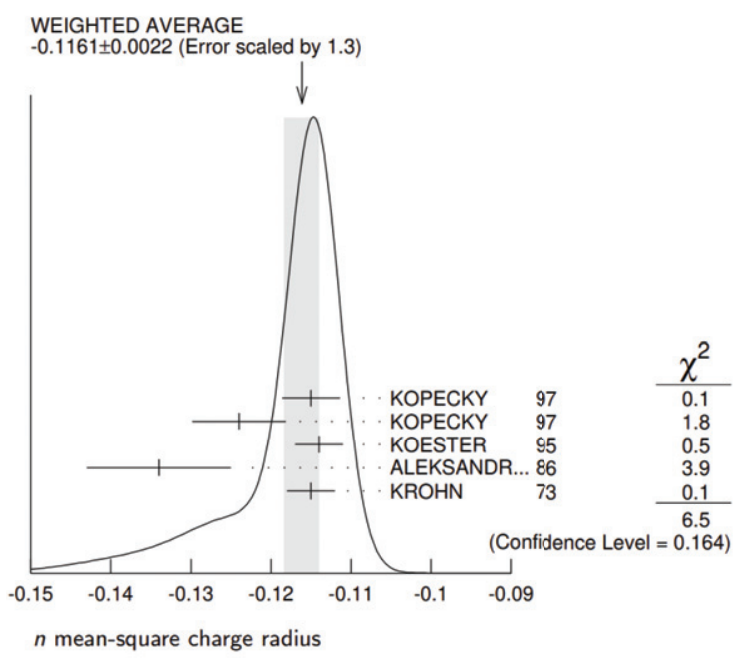

Fig. 3: Experimental determinations of the square of the neutron charge radius. Figure from [7].

The quark charges are in units of the elementary charge $e=1.602176634 \cdot 10^{-19} \mathrm{C}[7]$. Generalising [35] to independent angular velocities, we consider $2 \mathrm{D}$ surfaces $\mathbf{x}$ parametrised by the two excited degrees of freedom $\theta_{1}$ and $\theta_{3}$ for our flavour cases

$$
\mathbf{x}\left(\theta_{1}, \theta_{3}\right)=\left(\begin{array}{l}
x \\
y \\
z
\end{array}\right)=\left(\begin{array}{c}
\left(c+a \cos e_{q} \theta_{1}\right) \cos \left(-\theta_{3}\right) \\
\left(c+a \cos e_{q} \theta_{1}\right) \sin \left(-\theta_{3}\right) \\
a \sin e_{q} \theta_{1}
\end{array}\right) .
$$

The same kind of embedding was used in [24] where a first edition of fig. 1 was presented. First, one calculates the metric components, then one can find the local Gaussian curvature $K$ at each point. The result for $c=a$ is

$$
K_{q}=\frac{\cos e_{q} \theta_{1}}{a e_{q}\left(a+a \cos e_{q} \theta_{1}\right)} .
$$

See appendix B for details. We have flavour curvature expectation values

$$
\left\langle K_{q}\right\rangle=\int_{0}^{\pi} \int_{0}^{\pi} \int_{0}^{\pi} R^{*} K_{q} R \mathrm{~d} \theta_{1} \mathrm{~d} \theta_{2} \mathrm{~d} \theta_{3}
$$

For the leading component $f_{011}$, we find $\left\langle K_{u}\right\rangle=$ $0.15582 \cdots \mathrm{fm}^{-2}$ and $\left\langle K_{d}\right\rangle=-1.32875 \cdots \mathrm{fm}^{-2}$. We interpret the mean square of the neutron charge radius as an average over three colours of the reciprocal of the sum of the average Gaussian flavour curvatures for one $u$ quark and two $d$ quarks,

$$
\left\langle r_{n}^{2}\right\rangle=\frac{1}{3} \cdot \frac{1}{\left\langle K_{u}\right\rangle+2\left\langle K_{d}\right\rangle} .
$$

The result $(-0.1075 \pm 0.0020) \mathrm{fm}^{2}$ shown in table 1 seems promising in comparison with the value $(-0.1161 \pm$ $0.0022)^{f m^{2}}$ from the Particle Data Group [7] and even more promising compared to $[4,5]$ and the recent [9]. Note that all components in table 1 yield surprisingly consistent 
values for their $\left\langle r_{n}^{2}\right\rangle$ contributions. This supports the idea of $\left\langle r_{n}^{2}\right\rangle$ as a well-defined entity of intrinsic origin. Our value is some standard deviations away from the PDG value [7]. However, experimental determinations have shown quite a variation throughout the years, see fig. 3, and we consider the matter ripe for further investigation.

Conclusion. - We assumed the neutron structure to be of intrinsic origin determined by a mass Hamiltonian on the intrinsic configuration space $U(3)$. This space has local curvatures of varying signs. We assumed the mean square neutron charge radius to be a manifestation of the average Gaussian curvature of quark flavour tracks - generated in scattering by the momentum form - and weighted by the square of the wave function. The value derived is intriguingly close to values established experimentally in various experiments. We look forward to further experimental investigations for which we think the time is ripe.

I thank the referee for the queries which helped clarify generators and assumptions. I thank the Technical University of Denmark for an inspiring working environment.

Appendix A: scattering length from potential depth. - The scattering length is defined in effective range theory [36]. Using the Wronskian theorem one can relate the phase shifts under scattering by two different potentials to the Wronskian of the solutions to the radial Schrödinger equation including the respective potentials

$$
\frac{\hbar^{2}}{2 m_{n}}\left[-\frac{\mathrm{d}^{2}}{\mathrm{~d} r^{2}}+V(r)+\frac{l(l+1)}{r^{2}}\right] y_{l}(r)=E y_{l}(r) .
$$

The shape of the potential $V$ was not known except that it was supposed to be of finite range. To compare experimental results the convention was [11] to model by square wells of various depths $V_{0}<0$ but all of range equal to the classical electron radius $r_{e}$

$$
V(r)= \begin{cases}V_{0}, & r \leq r_{e} \\ 0, & r>r_{e}\end{cases}
$$

We follow Messiah [36] and consider solutions $\hat{y}_{l}$ and $y_{l}$ for two different potentials. Asymptotically with $E=\hbar^{2} k^{2} /\left(2 m_{n}\right)$

$$
\begin{aligned}
& y_{l}(r) \sim \sin \left(k r-l \frac{\pi}{2}+\delta_{l}\right), \\
& \hat{y}_{l}(r) \sim \sin \left(k r-l \frac{\pi}{2}+\hat{\delta}_{l}\right) .
\end{aligned}
$$

The Wronskian theorem yields

$$
\int_{r_{1}}^{r_{2}} W\left(y_{l}, \hat{y}_{l}\right)=-\int_{r_{1}}^{r_{2}} \hat{y}_{l}(U-\hat{U}) y_{l} \mathrm{~d} r
$$

with $U=\frac{2 m_{n}}{\hbar^{2}} V$ and $\hat{U}=\frac{2 m_{n}}{\hbar^{2}} \hat{V}$. Asymptotically

$$
\lim _{r \rightarrow \infty} W\left(y_{l}, \hat{y}_{l}\right)=k \sin \left(\delta_{l}-\hat{\delta}_{l}\right)
$$

and thus ${ }^{5}$

$$
\sin \left(\delta_{l}-\hat{\delta}_{l}\right)=-\frac{2 m_{n}}{\hbar^{2} k} \int_{0}^{\infty} \hat{y}_{l}(V-\hat{V}) y_{l} \mathrm{~d} r
$$

The scattering length $b$ is defined from the phase shift $\delta_{0}$ in $S$-wave scattering at vanishing incident energy as

$$
b \equiv-\lim _{k \rightarrow 0} \frac{\delta_{0}}{k} .
$$

Choosing $\hat{V}=0$ there is no scattering and one has $\hat{\delta}_{l}=0$ and $\hat{y}_{l}=k r j_{l}(k r)$, where $j_{l}$ is a spherical Bessel function of order $l$. In so far as the scattering length is finite, the phase shift $\delta$ will decrease with energy $E \sim k^{2}$ because of decreasing wave number $k$ and we can substitute $\delta$ for $\sin (\delta)$ to get an integral representation of the scattering length [37] with $j_{0}(k r)=\frac{\sin (k r)}{k r}$,

$$
b=\left.\int j_{0}(k r) U(r) f_{0}(r)\right|_{k \rightarrow 0} r^{2} \mathrm{~d} r, \quad f_{0}(r)=\frac{y_{0}(r)}{r} .
$$

Ross and Shaw [36] solve this to give an implicit expression

$$
b=\frac{r_{e} I}{1+I}
$$

and have

$$
\left.f_{0}\right|_{k \rightarrow 0}=\left.e^{i \delta} \frac{\sin (k r+\delta)}{k r}\right|_{k \rightarrow 0},
$$

where

$$
I=\left.\left(\left.r_{e} f_{0}\left(r_{e}\right)\right|_{k \rightarrow 0}\right)^{-1} \int U f_{0}(r)\right|_{k \rightarrow 0} r^{2} \mathrm{~d} r .
$$

Using (A.7) and a power expansion of the sine we have $[37]$

$$
\left.f_{0}\right|_{k \rightarrow 0}=\left.e^{i \delta} \frac{\sin (k r+\delta)}{k r}\right|_{k \rightarrow 0}=1-\frac{b}{r} .
$$

We insert the last expression in (A.10) and find

$$
I=\frac{1}{r_{e}-b} \int_{0}^{\infty} U(r)\left(1-\frac{b}{r}\right) r^{2} \mathrm{~d} r=\frac{2 m_{n} V_{0}}{\hbar^{2}} \frac{\frac{1}{3} r_{e}^{3}-\frac{1}{2} b r_{e}^{2}}{r_{e}-b}
$$

Solving (A.9) for the scattering length we get for $|b| \ll r_{e}$

$$
b \approx \frac{1}{3} r_{e}^{3} \frac{2 m_{n}}{\hbar^{2}} V_{0}
$$

The implied proportionality between $b_{n e}$ and $V_{0}$ agrees with the conversion used by Krohn and Ringo [8] although they do not state the above relation explicitly.

\footnotetext{
${ }^{5}$ Provided that $V$ and $\hat{V}$ go quicker than $1 / r$ to zero at infinity and have no singularity stronger than $1 / r^{2}$ at $r=0$ [36]. These requirements are met by the square well potentials we consider here.
} 
Appendix B: metric components for curvature calculation. - We follow [35] and find the metric coefficients of the square of the line element $\mathrm{d} s$,

$$
\begin{aligned}
\mathrm{d} s^{2} & =E \mathrm{~d} \theta_{3}^{2}+F \mathrm{~d} \theta_{3} \mathrm{~d} \theta_{1}+G \mathrm{~d} \theta_{1}^{2}, \\
E & \equiv \mathbf{x}_{\theta_{3}} \cdot \mathbf{x}_{\theta_{3}}=h^{2}\left(a+a \cos e_{q} \theta_{1}\right)^{2}, \\
F & \equiv \mathbf{x}_{\theta_{3}} \cdot \mathbf{x}_{\theta_{1}}=0, \\
G & \equiv \mathbf{x}_{\theta_{1}} \cdot \mathbf{x}_{\theta_{1}}=a^{2} e_{q}^{2} .
\end{aligned}
$$

Using [35]

$$
K=\frac{-1}{\sqrt{E G}}\left[\left(\frac{(\sqrt{E})_{\theta_{1}}}{\sqrt{G}}\right)_{\theta_{1}}+\left(\frac{(\sqrt{G})_{\theta_{3}}}{\sqrt{E}}\right)_{\theta_{3}}\right]
$$

the result in (38) follows.

Irons [35] uses a non-singular embedding with two different torus radii $c$ and $a$ where $c>a$. In case the singularity at $\cos e_{q} \theta_{1}=-1$ in (38) is of concern one may choose $c=a+\epsilon$ and let $\epsilon \rightarrow 0$. For the average curvatures (39) the singularity does not matter since the set of singular points are of zero measure in the $\left(\theta_{1}, \theta_{2}, \theta_{3}\right)$ eigenangle space. Thus (38) integrates to final results (39) over the domain of integration.

\section{REFERENCES}

[1] Sears V. F., Phys. Rep., 141 (1986) 281.

[2] Koester L., Waschkowski W., Mitsyna L. V., Samosvat G. S., Prokofjevs P. and Tambergs J., Phys. Rev. C, 51 (1995) 3363.

[3] Kopecky S., Harvey J. A., Hill N. W., Krenn M., Pernicka M., Riehs P. and Steiner S., Phys. Rev. C, 56 (1997) 2229.

[4] Filin A. A., Baru V., Epelbaum E., Krebs H., MÖller D. and Reinert P., Phys. Rev. Lett., 124 (2020) 082501 (arXiv:1911.04877v2 [nucl-th] (5 May 2020)).

[5] Filin A. A., Möller D., Baru V., Epelbaum E., Krebs H. and Reinert P., Phys. Rev. C, 103 (2021) 024313 (arXiv:2009.08911v1 [nucl-th] (18 September 2020)).

[6] Atac H., Constantinou M., Meziani Z.-E., Paolone M. and Sparveris N., Eur. Phys. J. A, 57 (2021) 65 (arXiv:2009.04357v3 [nucl-ex] (18 September 2020)).

[7] Particle Data Group (Zyla P. A. et al.), Prog. Theor. Exp. Phys., 2020 (2020) 083C01.

[8] Krohn V. E. and Ringo G. R., Phys. Rev. D, 8 (1973) 1305 .

[9] Heacock B. et al., Pendellösung interferometry probes the neutron charge radius, lattice dynamics and fifth forces, arXiv 2103.05428v1 (9 March 2021).
[10] Trinhammer O. L., EPL, 133 (2021) 31001.

[11] Fermi E. and Marshall L., Phys. Rev., 72 (1947) 1139.

[12] Foldy L. L., Phys. Rev., 87 (1952) 693.

[13] Warner F. W., Foundations of Differentiable Manifolds and Lie Groups (Springer, New York) 1983, p. 36.

[14] Belushin M. A., Hammer H.-W. and Meissner U.-G., Phys. Rev. C, 75 (2007) 035202.

[15] Carlitz R. D., Ellis S. D. and Savit R., Phys. Lett. $B, 68$ (1977) 443.

[16] Niégawa A. and Kiang D., Phys. Rev. D, 15 (1977) 3488.

[17] Heisenberg W., Ann. Phys. (Berlin), 32 (1938) 20.

[18] Landau L. D. and Lifshitz E. M., The Classical Theory of Fields, Course of Theoretical Physics, Vol. 2, 4th edition (Elsevier Butterworth-Heinemann, Oxford) 2005, p. 97.

[19] Trinhammer O. L., EPL, 131 (2020) 31001.

[20] Sakurai J. J., Advanced Quantum Mechanics (AddisonWesley, Redwood City) 1967, p. 194.

[21] Merzbacher E., Quantum Mechanics (John Wiley and Sons, Inc., New York, London) 1961 pp. 226, 187.

[22] Foldy L. L., Phys. Rev., 83 (1951) 688.

[23] Foldy L. L., Phys. Rev., 87 (1952) 688.

[24] Trinhammer O. L., EPL, 102 (2013) 42002 (arXiv: 1303.5283v2 [physics.gen-ph] (22 April 2014)).

[25] Kogut J. B. and Susskind L., Phys. Rev. D, 11 (1975) 395.

[26] Wilson K. G., Phys. Rev. D, 10 (1974) 2445.

[27] Manton N. S., Phys. Lett. B, 96 (1980) 328.

[28] Uhlenbkeck G. E. and Goudsmit S. A., Naturwissenschaften, 13 (1925) 953.

[29] Schiff L. I., Quantum Mechanics, 3rd edition, (McGrawHill, Kogakusha) 1968, pp. 324, 236, 209.

[30] Goldstein H., Classical Mechanics, 2nd edition (Addison Wedley, Reading) 1980, p. 102.

[31] Dirac P. A. M., The Principles of Quantum Mechan$i c s$, 4th edition (Oxford University Press, Oxford) 1958, p. 144.

[32] Milnor J., Ann. Math. Stud., 51 (1963) 1.

[33] Trinhammer O. L. and Olafsson G., The Full LaplaceBeltrami operator on $U(N)$ and $S U(N)$, arXiv:mathph/9901002v2 (10 April 2012).

[34] Weyl H., The Classical Groups. Their Invariants and Representations, Princeton Landmarks in Mathematics (Princeton University Press, Princeton, NJ) 1966 p. 197.

[35] Irons M. L., The Curvature and Geodesics of the Torus, http://www.rdrop.com/ half/math/torus/torus . geodesics.pdf (17 November 2005).

[36] Messiah A., Quantum Mechanics, Vol. 1 (North Holland Publishing Company, Amsterdam) 1964, p. 404.

[37] Ross M. H. and Shaw G. L., Ann. Phys., 9 (1960) 391. 